\title{
Spectral hole-burning and carrier-heating dynamics in InGaAs quantum-dot amplifiers
}

Borri, Paola; Langbein, Wolfgang Werner; Hvam, Jørn Märcher; Heinrichsdorff, F.; Mao, M.-H.; Bimberg, D.

Published in:

I E E E Journal on Selected Topics in Quantum Electronics

Link to article, DOI:

$10.1109 / 2944.865110$

Publication date:

2000

Document Version

Publisher's PDF, also known as Version of record

Link back to DTU Orbit

Citation (APA):

Borri, P., Langbein, W. W., Hvam, J. M., Heinrichsdorff, F., Mao, M-H., \& Bimberg, D. (2000). Spectral holeburning and carrier-heating dynamics in InGaAs quantum-dot amplifiers. I E E E Journal on Selected Topics in Quantum Electronics, 6(3), 544-551. https://doi.org/10.1109/2944.865110

\section{General rights}

Copyright and moral rights for the publications made accessible in the public portal are retained by the authors and/or other copyright owners and it is a condition of accessing publications that users recognise and abide by the legal requirements associated with these rights.

- Users may download and print one copy of any publication from the public portal for the purpose of private study or research.

- You may not further distribute the material or use it for any profit-making activity or commercial gain

- You may freely distribute the URL identifying the publication in the public portal 


\title{
Spectral Hole-Burning and Carrier-Heating Dynamics in InGaAs Quantum-Dot Amplifiers
}

\author{
P. Borri, Member, IEEE, W. Langbein, J. M. Hvam, F. Heinrichsdorff, M.-H. Mao, and Dieter Bimberg, Member, IEEE
}

\begin{abstract}
The ultrafast gain and index dynamics in a set of InAs-InGaAs-GaAs quantum-dot (QD) amplifiers are measured at room temperature with femtosecond resolution. The role of spectral hole-burning (SHB) and carrier heating $(\mathrm{CH})$ in the recovery of gain compression is investigated in detail. An ultrafast recovery of the spectral hole within $\sim 100$ fs is measured, comparable to bulk and quantum-well amplifiers, which is contradicting a carrier relaxation bottleneck in electrically pumped QD devices. The CH dynamics in the QD is quantitatively compared with results on an InGaAsP bulk amplifier. Reduced $\mathrm{CH}$ for both gain and refractive index dynamics of the QD devices is found, which is a promising prerequisite for high-speed applications. This reduction is attributed to reduced free-carrier absorption-induced heating caused by the small carrier density necessary to provide amplification in these low-dimensional systems.
\end{abstract}

Index Terms-Quantum-dot lasers, ultrafast optics.

\section{INTRODUCTION}

$\mathbf{S}$ ELF-ORGANIZED semiconductor quantum dots (QD's) based on InGaAs are one of the best systems available for fabrication of QD lasers [1]. These systems have strong confinement energies much higher than $k T$ even at room temperature (RT), well-defined interfaces as a consequence of the epitaxial growth, avoiding problems with surface states present in colloidal QD's embedded in a matrix, and emission in the infrared region suitable for applications in optical communication. Significant progress in the fabrication of In(Ga)As-GaAs QD lasers both with molecular beam epitaxy (MBE) and metalorganic chemical vapor deposition (MOCVD) have been recently achieved [1]. Ground-state lasing at RT, high internal quantum efficiency, and low threshold current density have already been demonstrated in both stacked-layer [2]-[5] and single-layer QD lasers [6], [7]. Efficient [8] and narrow-linewidth [9] RT emission at 1.3- $\mu \mathrm{m}$ wavelength has been achieved recently, and the first QD lasers operating in this wavelength region are already reported [10]-[13]. Other properties, like differential gain, small signal modulation bandwidth, and alpha parameter, are still under discussion in the comparison between the expected superior performances and the measured values.

Manuscript received January 13, 2000; revised March 29, 2000. This work was supported by the Danish Technical Research Council in the framework of SCOOP and by DFG in the framework of SFB 296.

P. Borri and J. M. Hvam are with the Research Center COM, The Technical University of Denmark, DK-2800 Lyngby, Denmark.

W. Langbein is with the Lehrstuhl für Experimentelle Physik EIIb, Universität Dortmund, 44221 Dortmund, Germany.

F. Heinrichsdorff, M.-H. Mao, and D. Bimberg are with the Institut für Festkörperphysik, Technische Universität Berlin, 10623 Berlin, Germany.

Publisher Item Identifier S 1077-260X(00)05099-1.
In order to understand the performance of QD-based devices, an investigation of the intrinsic properties of QD's is necessary. For example, it has been conjectured that carrier relaxation limits the modulation bandwidth in QD lasers [14], [15]. Previous investigations used mainly time-resolved photoluminescence spectroscopy on unprocessed thin film samples (see [16]-[19] and references therein). Beyond relaxation oscillation experiments [4], [15] direct time-resolved measurements of carrier dynamics in QD devices under electrical injection are not yet reported.

Optical pump-probe experiments have shown to provide direct information on the ultrafast dynamics in bulk and quantum-well semiconductor optical amplifiers (SOA's) [20]-[27]. In these previous reports, the subpicosecond gain and refractive index dynamics of the SOA's were measured. An ultrafast gain compression caused by stimulated emission induced by the pump pulse [spectral hole-burning (SHB)], which recovers by carrier-carrier scattering in less than $100 \mathrm{fs}$, and a slower recovery of the gain compression in the picosecond time scale caused by carrier heating $(\mathrm{CH})$ were observed [24], [25].

In this work, we have applied the pump-probe technique to a set of InAs-InGaAs-GaAs amplifiers of different geometries. We investigate in detail the role of SHB and $\mathrm{CH}$ for the gain dynamics of QD amplifiers. For a quantitative discussion, the measured dynamics is compared with results obtained on a commercial InGaAsP bulk SOA operating at $1.53 \mu \mathrm{m}$. We find that the SHB in the QD amplifier has an ultrafast recovery similar to the bulk case, showing a fast carrier relaxation also in electrically pumped QD's. The CH dynamics, however, is different in the two cases. CH significantly affects the recovery of gain compression in the bulk SOA. In QD amplifiers, gain compression is dominated by the ultrafast SHB with a minor role of $\mathrm{CH}$, making devices based on such structures very promising for high-speed applications. The paper is organized as follows: the description of the investigated samples and the experimental setup is provided in Section II. The transmission properties measured for the different QD devices are described in Section III. In Section IV, the pump-probe measurements are shown and the role of SHB for the QD gain dynamics is discussed using a quantitative fit procedure. In Section V, the $\mathrm{CH}$ dynamics is discussed and compared with the bulk case. Section VI gives a summary of the results.

\section{SAMPLES AND EXPERIMENT}

The QD samples are p-type-intrinsic-n-type (p-i-n) structures grown by MOCVD. The active region consists of three stacked layers of binary/ternary InAs-InGaAs QD's separated by 21-nm-thick GaAs barriers, placed in the center of a $120-\mathrm{nm}$ 


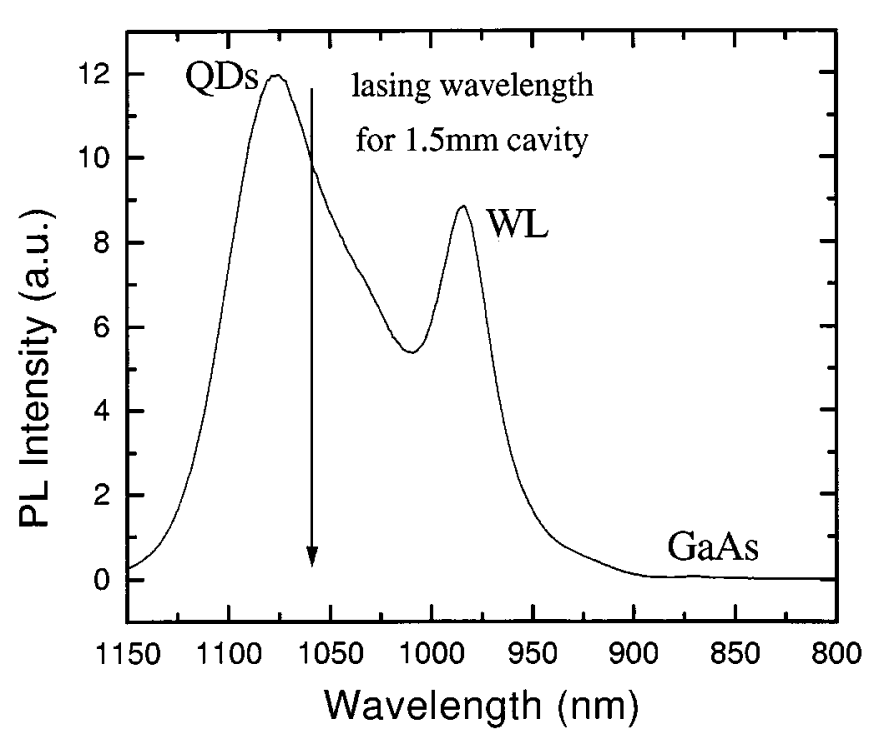

Fig. 1. Room-temperature PL after etching off the top cladding. The QD distribution and the wetting layer transition are clearly resolved.

GaAs layer. Two $\mathrm{Al}_{0.7} \mathrm{Ga}_{0.3} \mathrm{As}$ cladding layers and a ridge structure provide optical confinement and waveguiding [3]. The samples had ridge widths of 6 and $8 \mu \mathrm{m}$ and lengths of $0.26,0.475$, and $1.01 \mathrm{~mm}$. The end facets were tilted, avoiding backreflection into the waveguide mode. This inhibited lasing and allowed for single-pass amplification measurements. The photoluminescence spectrum at RT after etching of the top cladding is shown in Fig. 1. The spectrum shows a ground-state dot transition at $1.148 \mathrm{eV}(1.08 \mu \mathrm{m})$ with a broadening of about $60 \mathrm{meV}$, and a wetting layer transition separated by $110 \mathrm{meV}$. Laser action at RT in the ground-state dot transition of this type of structure was demonstrated [3]. For 1.5-mm length with normal facets, the investigated structure showed RT lasing at $1.057-\mu \mathrm{m}$ wavelength with a threshold current density [28] of $417 \mathrm{~A} / \mathrm{cm}^{2}$. The bulk device investigated for comparison is a commercial InGaAsP-InP SOA with tilted and antireflection-coated facets. It has a $3-\mu \mathrm{m} \times 250-\mu \mathrm{m}$ ridge waveguide and operates at $1.53 \mu \mathrm{m}$, with a maximum small signal gain of $13 \mathrm{~dB}$ at $120-\mathrm{mA}$ bias current.

The gain of the devices was measured using the transmission of a short laser pulse through the device. The laser source was the idler of an optical parametric amplifier pumped by a $\mathrm{Ti}$ : sapphire regenerative amplifier system, producing $150-\mathrm{fs}$ pulses tunable from 0.9 to $2.5 \mu \mathrm{m}$ at a $300-\mathrm{kHz}$ repetition rate. Linear chirp compensation of the laser pulse is achieved with a pulse shaper, providing nearly Fourier-limited pulses. The light is coupled into and out of the device by high numerical-aperture aspheric lenses, and detected by a thermoelectrically cooled $\mathrm{Ge}$ detector with lock-in technique. The ultrafast gain and index dynamics were measured with a pump-probe experiment using an heterodyne detection scheme similar to earlier measurements on bulk and quantum-well amplifiers [24], [25]. We have recently demonstrated a novel version of this detection scheme, suitable for low repetition rate laser systems of large $(10 \%)$ amplitude noise. Details of the setup can be found in [29]. In all of the measurements, linear polarization corresponding to the transverse electric (TE) waveguide mode was used. a)
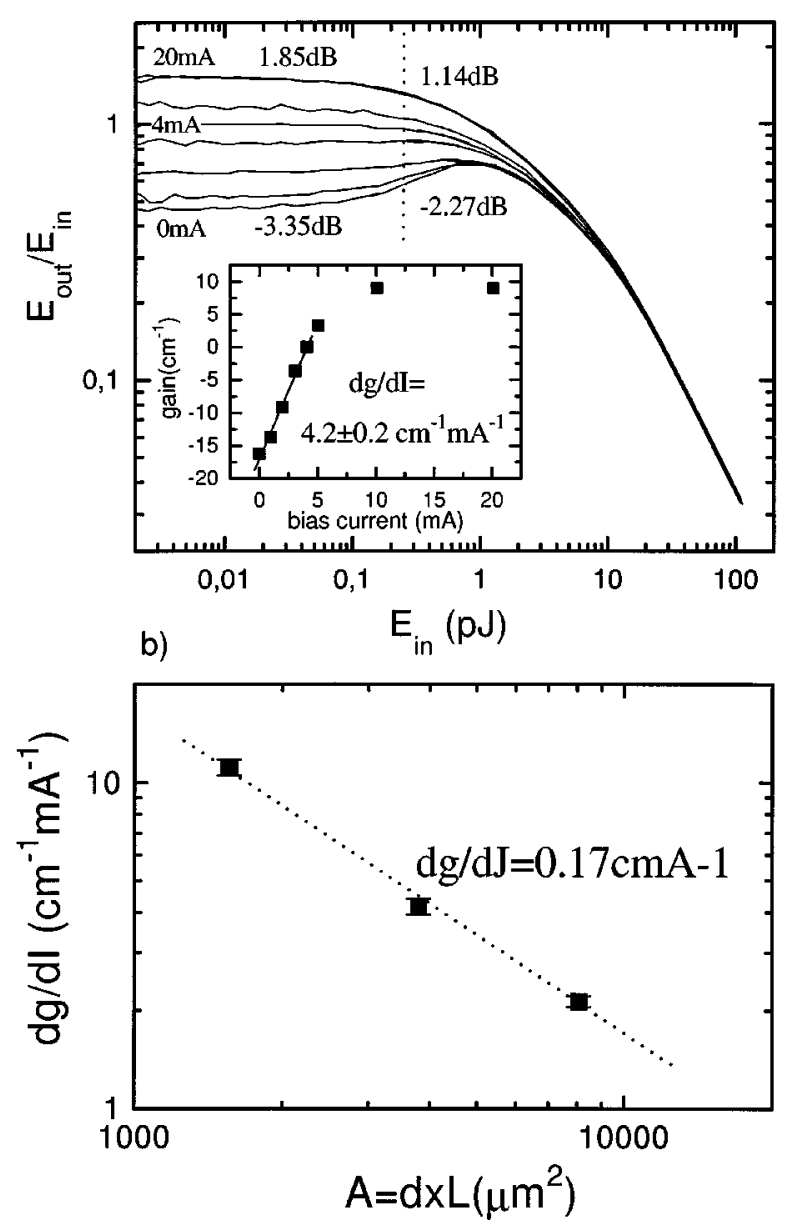

Fig. 2. (a) Device gain measured from the transmission of a 150 -fs optical pulse through a 8- $\mu \mathrm{m} \times 475-\mu \mathrm{m}$ QD amplifier versus the input pulse energy for different bias currents, as indicated. The maximum gain and absorption values are indicated. In the inset, the corresponding modal gain and a linear fit to the modal gain versus current before saturation are shown. (b) Differential gain versus area factor, defined as the product of the device length $L$ times the ridge width $d$. A constant derivative of the gain with respect to current density is deduced for devices of different geometries.

\section{TRANSMISSION PROPERTIES}

The device gain $G=e^{g L}$, with the modal gain $g$ and the length of the device $L$, is deduced from the transmission of 150 -fs pulses centered at $1.08 \mu \mathrm{m}$ at different bias currents, as shown in Fig. 2(a) for the 8- $\mu \mathrm{m} \times 475-\mu \mathrm{m}$ QD device. For small input pulse energies, an absorption of $-3.35 \mathrm{~dB}$ is found at zero bias, transparency occurs at $4 \mathrm{~mA}$ (corresponding to a current density of $J_{t r}=105 \mathrm{Acm}^{-2}$ ), and a maximum gain of $1.85 \mathrm{~dB}$ is reached at $10 \mathrm{~mA} .{ }^{1}$ From the bias dependence of the modal gain [see inset of Fig. 2(a)], a differential gain $d g / d I=4.2$ $\mathrm{cm}^{-1} \mathrm{~mA}^{-1}$ before saturation is deduced. Amplified sponta-

\footnotetext{
${ }^{1}$ Note that the transparency current is deduced from the pump-probe measurements, as will be shown in the following, and corresponds to the case when the inversion of population is zero and $g=0$. The effective transmission (ratio between the pulse energy at the output and at the input of the device) is additionally affected by the waveguide losses. Waveguide losses of $9.9 \mathrm{~cm}^{-1}$ were estimated in these structures [4]. The input pulse energy in Fig. 2(a) is deduced from the measured output pulse energy and the device gain, neglecting waveguide losses, and should be increased by a factor 1.6 for $9.9-\mathrm{cm}^{-1}$ losses. This is also in agreement with the pulse energy measured before the input aspheric lens and the estimated input coupling efficiency.
} 


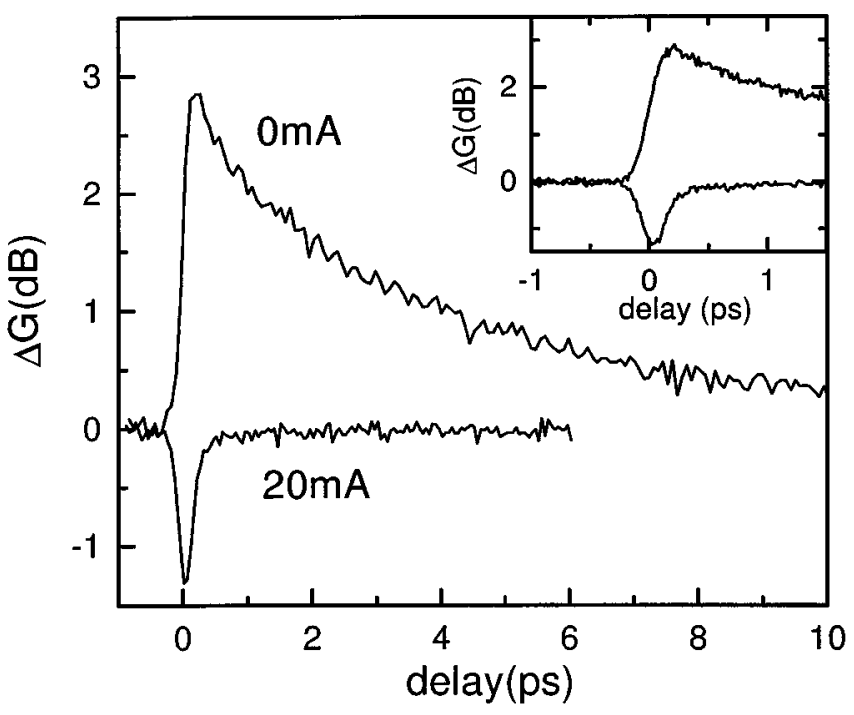

Fig. 3. Pump-induced gain change measured in a heterodyne pump-probe experiment at maximum gain $(20 \mathrm{~mA})$ and absorption $(0 \mathrm{~mA})$ in the $8-\mu \mathrm{m} \times$ $475-\mu \mathrm{m}$ QD device. In the inset, a zoom of the dynamics up to $1.5 \mathrm{ps}$.

neous emission spectra show the saturation of the ground-state dot transition and the occupation of excited states above $10 \mathrm{~mA}$, in agreement with the saturation of the gain. Similar measurements were performed on a $6-\mu \mathrm{m} \times 260-\mu \mathrm{m}$ and a $8-\mu \mathrm{m} \times$ $1010-\mu \mathrm{m}$ device. The differential gain versus the area factor in the three samples is shown in Fig. 2(b). Within error bars, a constant derivative of the modal gain with respect to the current density $\left(d g / d J=0.17 \mathrm{~A}^{-1} \mathrm{~cm}\right)$ is deduced, independent of the amplifier geometry, thus representing the "intrinsic" differential gain of the QD structure. Measurements performed for comparison on the commercial InGaAsP bulk amplifier show a value of $d g / d J=0.023 \mathrm{~A}^{-1} \mathrm{~cm}$ around transparency, a factor of 7.4 smaller than that for the QD devices, and a transparency current density of $J_{t r}=4670 \mathrm{Acm}^{-2}$. Schönfelder et al. [30] recently reported the differential gain in high-speed GaAs-based multiple quantum-well lasers, with an optimum value around threshold of $d g / d I=13.32 \mathrm{~cm}^{-1} \mathrm{~mA}^{-1}$ at $1.092 \mu \mathrm{m}$ on a $3-\mu \mathrm{m} \times 200-\mu \mathrm{m}$ laser containing four InGaAs-GaAs quantum wells, which corresponds to $d g / d J=0.080 \mathrm{~A}^{-1} \mathrm{~cm}$, a factor 2.1 smaller than in the investigated QD devices.

With increasing input energy, a bleaching of the absorption or a depletion of the gain occurs because of stimulated transitions induced by the pulse, as shown in Fig. 2(a). For high input energies $(>1 \mathrm{pJ})$, an additional reduction of the transmission from two-photon absorption (TPA) is present at all bias currents. For a pump input energy of $0.27 \mathrm{pJ}$, which is in the small signal regime [see dotted line in Fig. 2(a) and the indicated transmission values], the induced change in the probe transmission has been investigated by the time-resolved pump-probe experiment, as reported in the next section.

\section{GAIN AND INDEX DynAmiCs: SPECTRAL Hole-BURNING}

In Fig. 3, the gain change in decibels deduced from the probe transmission change is shown for a bias of 0 and $20 \mathrm{~mA}$, respectively, as a function of the pump-probe delay, for a pump input energy of $0.27 \mathrm{pJ}$ and a probe energy ten times smaller.
A striking difference between the absorption and the gain recovery dynamic appears. At $0 \mathrm{~mA}$, pump-induced absorption bleaching occurs, which reaches a maximum value of $2.8 \mathrm{~dB}$ and then recovers over several picoseconds. At 20-mA bias, a gain compression up to $-1.3 \mathrm{~dB}$ that recovers in less than $0.3 \mathrm{ps}$ is observed. The data show that the pump-induced SHB in the absorption case builds with the time integral of the pulse, according to a recovery time much longer than is the pulse duration, whereas in the gain case, it recovers on a time comparable or shorter than is the pulse duration. In the inset, a zoom of the dynamics over the first $1.5 \mathrm{ps}$ is shown. It can be clearly seen that the maximum absorption bleaching occurs after the pump pulse, whereas the gain compression nearly follows the pulse intensity autocorrelation. This is also reflected in the higher maximum absorption bleaching compared with the gain compression.

These dynamics are interpreted in the following physical picture. In the absorption case, the dots are initially empty. When the pump arrives, electron-hole pairs (excitons) are created in the dot ground state, leading to a bleaching of the absorption. This recovers by spontaneous carrier recombination or by carrier escape from the dot ground state. Typical spontaneous lifetimes in QD's are of the order of several hundreds of picoseconds, as measured by time-resolved photoluminescence [19], much longer than the measured recovery of the absorption bleaching. We thus attribute the recovery to thermally activated carrier escape, in agreement with recent experiments [31]. In Fig. 4, a fit to the data is shown, which is obtained by convoluting the pulse intensity autocorrelation with the material response function [24], [32]. We obtain a good fit using a biexponential response function with two equally weighted time constants of $1.25 \pm 0.03$ ps and $5.9 \pm 0.1 \mathrm{ps}$. They can be attributed to hole and electron escape times, because the absorption bleaching is proportional to the sum of electron and hole occupation numbers [16], [33]. The escape, which represents a heating of the optically excited cold excitons, also appears in the phase dynamics (see inset of Fig. 4). In fact, a heating of the carrier distribution results in an increase of the refractive index and, therefore, of the measured phase change [24]. In the gain case, the dots are initially occupied and, by stimulated emission, the pump leads to a reduction of the exciton population (gain compression) that recovers by relaxation of carriers into the dot ground state. The measured recovery time is, therefore, a direct measure of the carrier relaxation time from excited states into the dot ground state in a QD amplifier under working conditions. Note that the used bias current of $20 \mathrm{~mA}$ corresponds to a maximum modal gain that is just enough for ground-state lasing of the investigated structure, and it is a realistic working point for QD lasers.

One of the major difficulties in interpreting quantitatively gain dynamics in SOA's is given by the occurrence of additional contributions to the probe transmission changes, like TPA and coherent artifacts [24], [34]. The TPA of the probe and pump pulses results in a reduced probe transmission that follows the intensity autocorrelation and therefore overlaps with the SHB gain compression [24]. Especially in low-active-volume devices, like quantum-well and QD SOA's, the nonresonant TPA contribution from the barrier or the cladding region relative to the SHB from the active region can be sizeable [24]. 


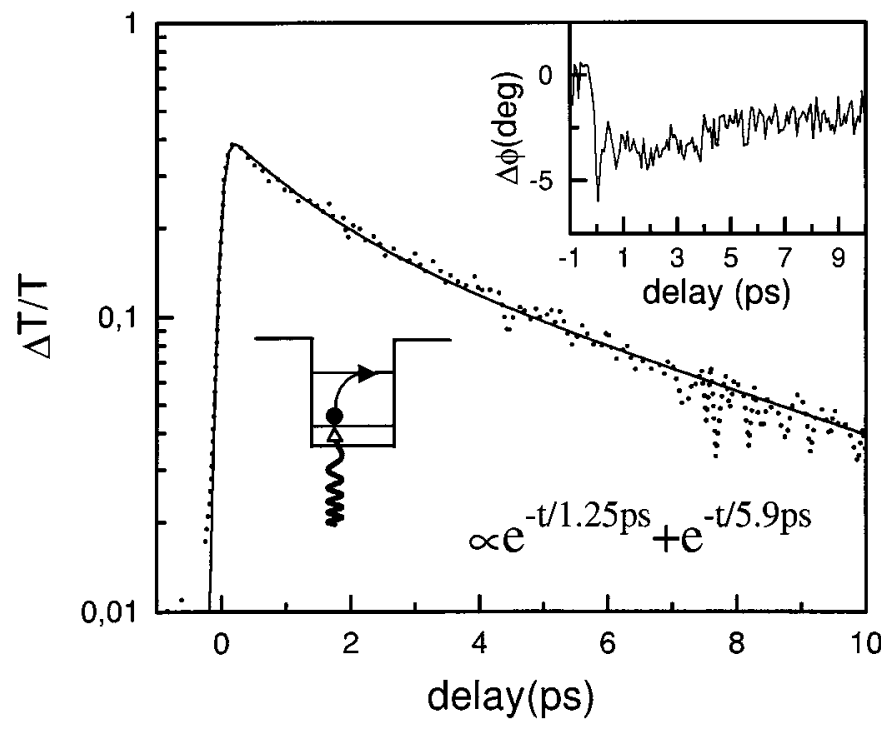

Fig. 4. Differential probe transmission change in absorption $(0 \mathrm{~mA})$ versus delay time. The solid line is a biexponential fit to the data proportional to the indicated formula. The inset shows the corresponding phase change. A sketch of the process is shown in the figure: when the pump photon arrive (curly arrow), excitons are created in the dot ground state and absorption bleaching occurs. This recovers via phononassisted escape out of the dot ground state. The two exponential recovery times correspond to hole and electron escape times.

Because we measure a TPA independent of the bias current (i.e., mainly not in the active region), according to Fig. 2, we can estimate this contribution by comparison with the differential transmission at the transparency current, where the net stimulated transition rate is zero and only the TPA effect should appear. In Fig. 5, we show the gain dynamics at 4 and $20 \mathrm{~mA}$ for three increasing pump energies. The reduction in the probe transmission at $4 \mathrm{~mA}$ scales linearly with the pump energy (when corrected for the small absorption bleaching still present) as expected by TPA, whereas the gain change is sublinear according to the saturation of the SHB. Thus, the TPA strongly influences the gain dynamic only at higher input energies, and it is still a small contribution at $0.27 \mathrm{pJ}$, which we estimated to $-0.5 \mathrm{~dB}$ at zero delay (see also the fit in Fig. 5). The fit at $20 \mathrm{~mA}$ is then performed by fixing the amplitude of the TPA contribution, and including the so-called coherent artifact as half of the signal at zero pump-probe delay, as predicted for copolarized pump and probe [34]. The amplitude of the SHB effect is given by comparison with the maximum absorption bleaching that provides the strength of the stimulated transitions induced by the pump (scaled by the measured gain/absorption ratio). The only free parameter left is the SHB recovery time constant, and we obtain from the best fit, $115 \pm 10 \mathrm{fs}$, slightly shorter than is the pulse duration. Note that the fitting procedure is based on a convolution of the pulse intensity autocorrelation with a response function. The measured recovery time of the response function, thus, can be shorter than is the pulse duration, resulting in a small, but measurable, shift and asymmetry toward positive delay, as observed.

The SHB recovery in QD's is thus comparable with the one measured in bulk and quantum-well amplifiers [24], [25]

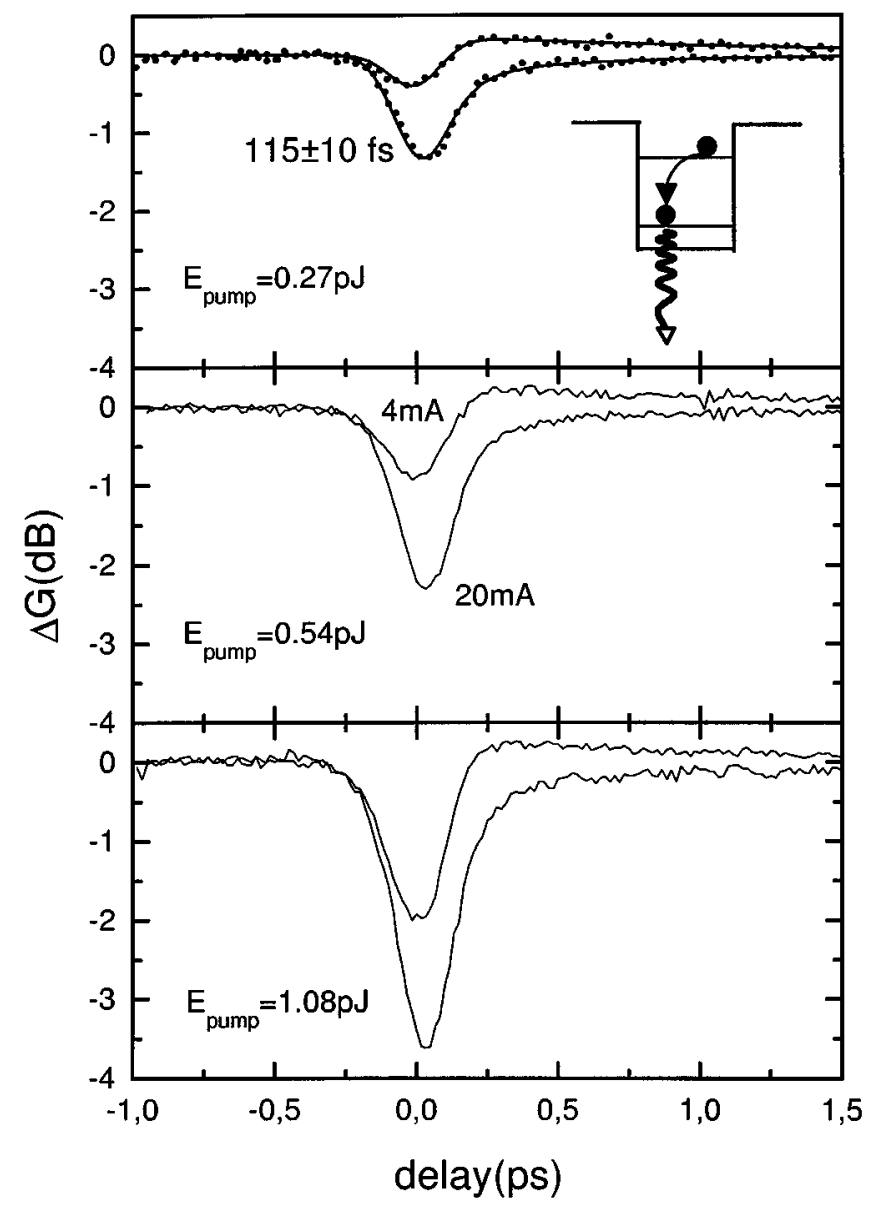

Fig. 5. Gain dynamics at 4 and $20 \mathrm{~mA}$ for different pump energies, as indicated. In the top part, the data are shown in dotted lines and the solid lines are fits. The fit at $4 \mathrm{~mA}$ is obtained using $-0.5-\mathrm{dB}$ TPA amplitude at zero delay plus a small absorption bleaching. A sketch of the process is shown in the figure: when the pump photon arrive (curly arrow), stimulated emissions remove excitons from the dot ground state and reduce the gain. This recovers via relaxation of carriers from the excited states into the dot ground state.

and indicates the lack of any relevant relaxation bottleneck in electrically pumped QD's, very promising for high-speed applications. The carrier relaxation corresponding to the SHB recovery occurs probably via Auger scattering from the dot excited states, partly occupied at $20 \mathrm{~mA}$, into the dot ground state, as recently also theoretically treated [35], [36]. Note that the occurrence of such ultrafast scattering processes imply fast dephasing times, i.e., large homogeneous broadening (10-20 meV), which we have confirmed by four wave-mixing experiments [37], [38]. Recent improvements in the growth quality have reduced size and alloy nonuniformities in InGaAs QD distributions, leading to linewidths as narrow as [9] $21 \mathrm{meV}$, comparable to the measured homogeneous broadening. Therefore, the common opinion of QD lasers being dominated by inhomogeneous broadening of the gain medium has to be revised. In fact, RT QD laser performances influenced by the homogeneous broadening, useful for continuous-wave single-mode lasing, have been recently reported [39].

A key parameter in the ultrafast SHB recovery is the electrical injection. This is already suggested by the comparison with the much slower absorption recovery dynamic observed 


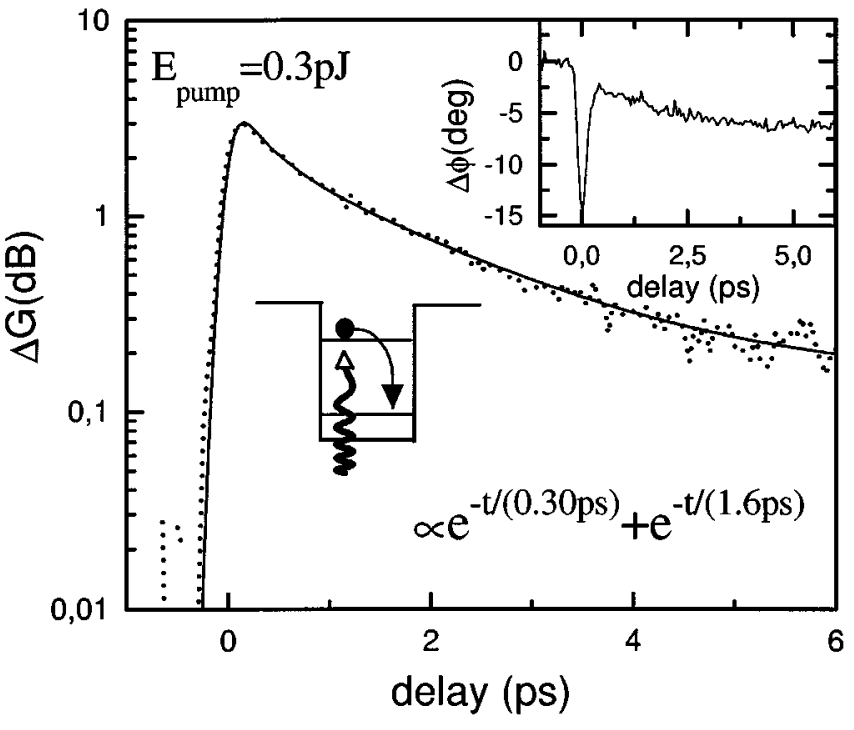

Fig. 6. Absorption bleaching recovery at $0 \mathrm{~mA}$ for pump and probe pulses at $1.04-\mu \mathrm{m}$ wavelength. The solid line is a biexponential fit to the data proportional to the indicated formula. The inset shows the corresponding phase change. A sketch of the process is shown in the figure: when the pump photon arrive (curly arrow), excitons are created in the dot excited states and absorption bleaching of the excited states occurs. This recovers via phononassisted relaxation of carriers into the dot ground state. The two exponential recovery times correspond to hole and electron relaxation times.

at $0 \mathrm{~mA}$, governed by phonon-assisted escape in absence of carrier-carrier scattering because of the lack of electrically injected carriers. Note that in bulk and quantum wells, the SHB recovery is also ultrafast in the absorption case, which is always investigated in the presence of a bias current [24]. In Fig. 6, we show the recovery dynamics at $0 \mathrm{~mA}$ when optically exiting and probing at $1.04 \mu \mathrm{m}$, i.e., in the dot excited-state transitions (see Fig. 1). The observed dynamics corresponds to the recovery of the excited states, initially occupied via the absorption of the pump, which occurs via carrier escape or via relaxation into the dot ground state. The phase dynamics (see inset of Fig. 6) shows a carrier cooling, instead of the heating observed in Fig. 4, thus indicating a dominant relaxation of the excited carriers into the dot ground state, rather than the promotion into high-energy states via escape. From the fit of these data, we measure a biexponential recovery time with $0.3 \pm 0.01$ and $1.6 \pm 0.05 \mathrm{ps}$, independent on the pump energy within one order of magnitude variation. This can be attributed to hole and electron phonon-assisted relaxation times into the dot ground state, when neglecting the carrier escape. More than likely, these times are controlled by the mixture of the two processes, relaxation and escape, and are thus lower limits to the relaxation times. These results show that the phonon-assisted relaxation dynamics is slower than is the ultrafast SHB recovery shown in Fig. 5, which supports the interpretation that Auger scattering in presence of electrically injected carriers is responsible for the measured ultrafast SHB recovery.

Note that the influence of the bias current on the ultrafast SHB recovery is different in the QD SOA's compared with bulk or quantum-well SOA's. As mentioned above, in bulk and quantum wells, the recovery of the SHB is also ultrafast in the absorption case, in the presence of bias current. This is not the case in the QD SOA's, where only for a bias current above transparency is the ultrafast recovery of the SHB observed. In the next section, the bias dependence of the gain dynamics is shown for the 1.01-mm-long device (see Fig. 8). For a bias current of half the transparency current, a long recovery time of the absorption bleaching is still observed. We believe that, because of the discrete nature of the occupation in QD's and their spatial separation, the presence of bias current corresponding to less than one electron-hole pair per dot in average (that is below transparency) cannot lead to efficient carrier-carrier scattering, differently from bulk and quantum-well SOA's. Above transparency, saturation of the dot ground-state transition and filling of the excited states is measured in the amplified spontaneous emission spectra. In this case, intradot Auger scattering between the carriers in the excited states, and consequent relaxation into the dot ground state, can occur and lead to the measured ultrafast recovery of the SHB in QD's.

\section{GAIN AND INDEX DYNAMICS: CARRIER HEATING}

A surprising aspect of the measured gain dynamics in the QD device (see Fig. 3) is the lack of significant $\mathrm{CH}$ effects in the gain case, compared with what is usually observed in bulk and quantum-well devices [24], [29]. From the fit to the gain data at large delay, we can recover a minor contribution with a slower recovery time of $0.7 \pm 0.1 \mathrm{ps}$. In order to investigate quantitatively this aspect, we have compared the results on the QD device with the ones on a commercial InGaAsP bulk SOA.

In Fig. 7, we compare the gain dynamics measured on the bulk device at the maximum achievable small signal gain $(13 \mathrm{~dB})$ with the gain dynamics of the QD device at $20 \mathrm{~mA}$. For the case of the QD gain dynamics, we have subtracted the TPA contribution, in order to isolate the response from the active region. The TPA contribution to the gain dynamics in the bulk is negligible. The bulk gain dynamics is measured for a pump energy corresponding to $-6-\mathrm{dB}$ maximum gain compression, i.e., for the same ratio to the small signal gain, as in the dot case. In this way, we are comparing situations in which the pump-induced gain compression gives the same relative effect at zero delay, according to a comparable SHB response in the two materials. The data of the bulk device are then normalized to the data of the QD device, in order to compare the relative role of $\mathrm{CH}$ and SHB dynamics in the two cases. The bulk device shows a clear $\mathrm{CH}$ recovery on a ps time scale, significantly affecting the gain compression after the pump pulse. The QD device, instead, shows a much faster gain compression recovery, dominated by the SHB recovery. In Fig. 7(b), the phase dynamics of the QD device are also compared with the bulk data, normalized by the same factor used in Fig. 7(a). The phase dynamics are not sensitive to the SHB component [24] and show the $\mathrm{CH}$ recovery only. The phase of the QD device is also reduced compared with the bulk case, and it evolves on a longer time scale, which we have fitted with $2 \pm 0.5 \mathrm{ps}$. The reduced $\mathrm{CH}$ effect in both gain and phase dynamics of the QD device suggests superior speed performance and low-chirp operation.

The $\mathrm{CH}$ in the QD compared with the bulk is less reduced in the phase dynamics than it is in the gain dynamics. The maximum $\mathrm{CH}$ phase reduction is about a factor of two, whereas 


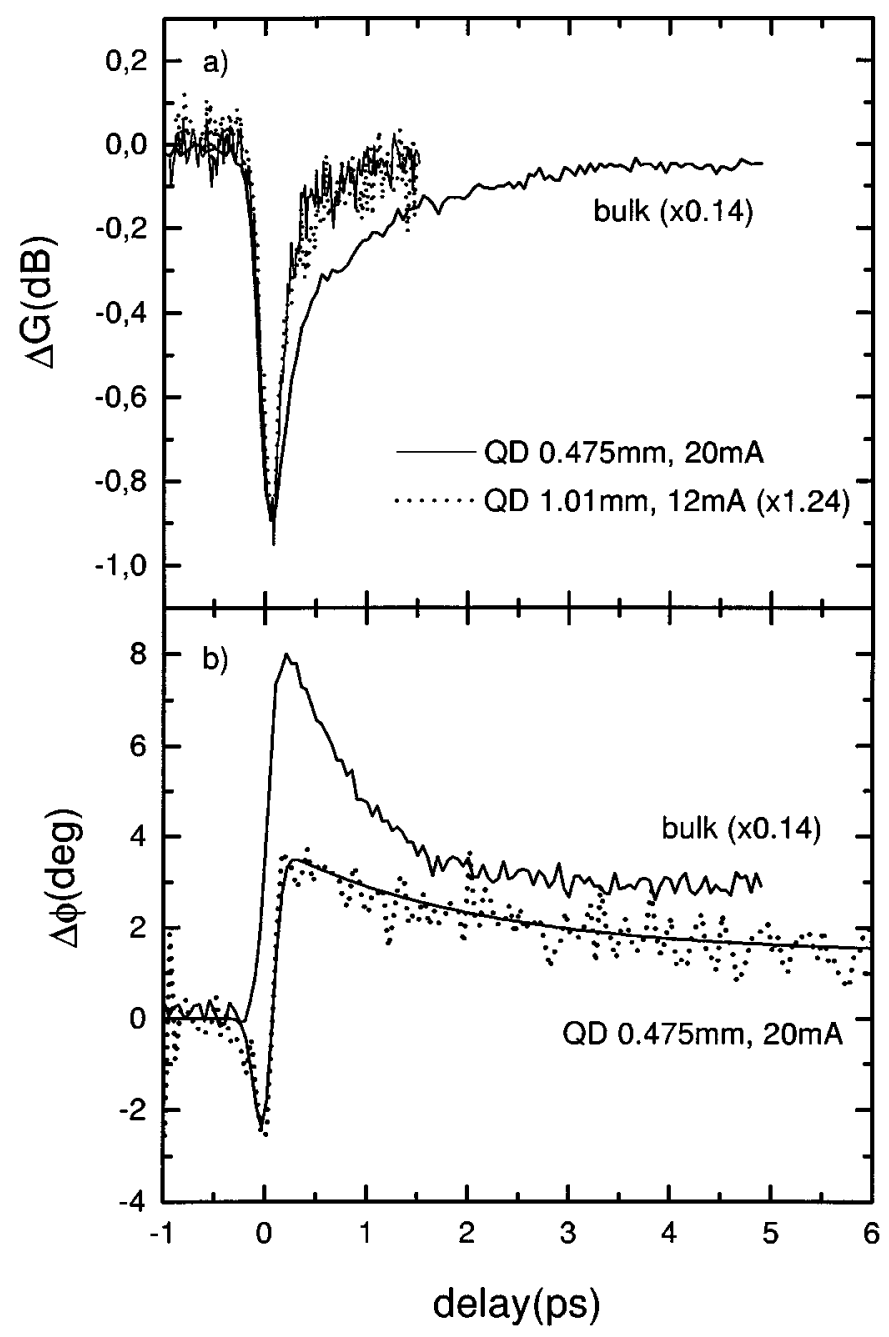

Fig. 7. Comparison of gain (a) and phase (b) changes in a bulk InGaAsP SOA and in the QD amplifiers. Reduced carrier heating in both gain and phase dynamics occurs in the QD devices.

directly after the pulse, the $\mathrm{CH}$ gain compression is about a factor of three smaller in the dots than it is in the bulk case. This could be from the gain saturation at $20 \mathrm{~mA}$. In fact, gain saturation indicates a strongly degenerate carrier distribution, with the Fermi level far above in energy. Also, in bulk and quantum-well devices, the gain dynamic are less sensitive to temperature effects far below the Fermi level, whereas the phase dynamics are sensitive to the nonresonant carrier distribution because the refractive index is related to the spectral integral of the gain via Kramers-Kronig transformations [24]. In order to investigate this point, we have performed pump-probe measurements on a 1.01-mm-long device, tuning the bias current from 0 to $12 \mathrm{~mA}$, within the linear part of the gain versus bias current. The results are shown in Fig. 8 for both the gain and the phase dynamics. Even when tuning the current just above transparency, the gain recovery dynamics is dominated by the ultrafast SHB component. In Fig. 7(a), we compare the gain dynamics at $12 \mathrm{~mA}$ (dotted line), after subtracting the TPA contribution inferred at $8 \mathrm{~mA}$, with the gain dynamics of the 0.475 - $\mathrm{mm}$-long device at $20 \mathrm{~mA}$ and the bulk device. The data have been normalized to the gain compression at zero delay. The carrier heating effect is

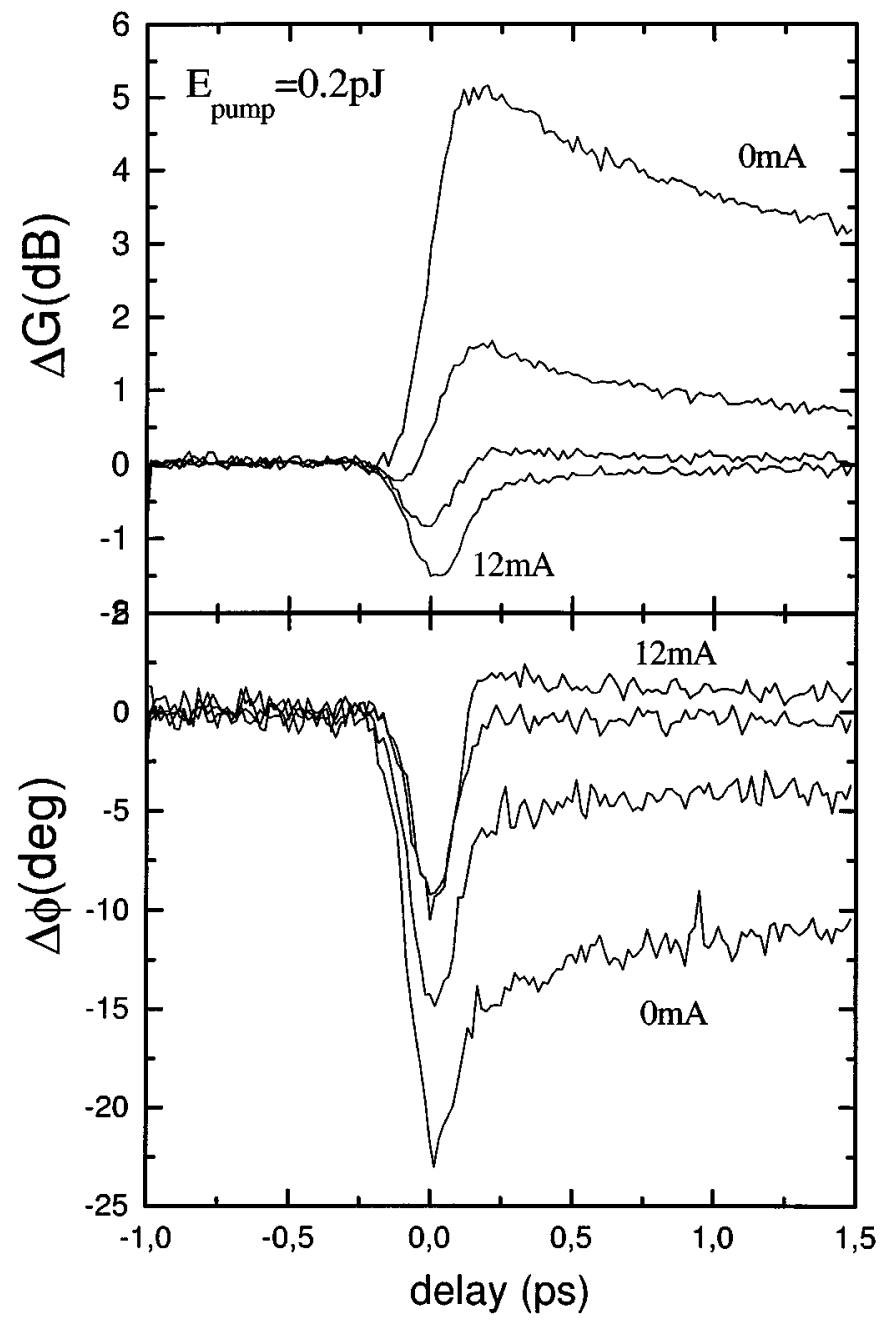

Fig. 8. Gain and phase dynamics in a 8- $\mu \mathrm{m} \times 1010-\mu \mathrm{m}$ QD amplifier, for bias current ranging from 0 to $12 \mathrm{~mA}$ in step of $4 \mathrm{~mA}$. Transparency occurs at $8 \mathrm{~mA}$.

still significantly smaller than it is in the bulk case, but slightly larger than in the 0.475-mm-long QD device, in agreement with the saturation argument. The $\mathrm{CH}$ in the phase dynamics, however, is found identical for the two QD devices, after scaling the phase data at $12 \mathrm{~mA}$ with the same factor used for the gain data, as indicated in Fig. 7(a).

Even if the saturation argument can explain a more pronounced effect of the reduced $\mathrm{CH}$ in the gain compared with the phase dynamics, we still observe an overall $\mathrm{CH}$ reduction in both gain and phase dynamics of the QD devices, also far from gain saturation. This is very promising for fast electrooptical applications that require operation at high differential gain. Besides the impact of the observed dynamics for device applications, we would like to comment on the possible physical origin of these effects.

Let us first recall what are the physical mechanisms responsible of carrier heating in bulk and quantum-well SOA's. CH occurs because of stimulated transitions, when cold carriers, below the average carrier energy, are removed from the distribution or hot carriers, above the average energy, are created [40], [24]. Conversely, stimulated transitions cool the distribution when cold carriers are created or hot carriers are removed [22]. Al- 
though these effects vanish at transparency, a significant $\mathrm{CH}$ is usually still observed in bulk and quantum-well SOA's [24], [29]. This is because of free carrier absorption (FCA) heating (or TPA heating at high pump powers [23]), in which carriers of a high excess of energy are created in the conduction and valence bands. The FCA heating often dominates over the stimulated transitions in bulk and quantum wells, resulting in a net heating in all operation regimes (gain, transparency, and absorption) of the SOA [24].

The measured phase dynamics in the dot devices change sign across transparency, as shown in Fig. 8 for the 1.01-mm device and in Figs. 4 and 7 for the $0.475-\mathrm{mm}$ device. At transparency, we do not measure any significant FCA heating. The heating dynamics in the QD devices are thus dominated by stimulated transition effects, different from bulk and quantum wells. We believe that the FCA heating is reduced in QD's compared with bulk and quantum wells because of the much lower total carrier density necessary to invert these structures. We conclude, therefore, that in the QD SOA's, the FCA is reduced compared with bulk and quantum wells, leading to a reduction of carrier heating in both gain and index dynamics, where mainly stimulated transition heating or cooling appears. The longer heating recovery time in the phase dynamics of QD's compared with the bulk [see Fig. 7(b)] is thus probably caused by the different physical mechanism governing the heating dynamics in the two materials.

\section{SUMMARY}

In summary, we have measured the transmission properties and the ultrafast gain and index dynamics on the dot groundstate transition in InAs-InGaAs-GaAs QD amplifiers. A differential gain $d g / d J=0.17 \mathrm{~A}^{-1} \mathrm{~cm}$ is measured, higher than in bulk and quantum-well devices. An ultrafast SHB recovery of $\sim 100$ fs dominates the gain compression dynamics, as quantitatively inferred from a fitting procedure in which the role of additional nonlinearities, such as TPA and coherent artifacts, was taken into account. The ultrafast SHB recovery is strictly related to the use of bias current, as demonstrated by comparing the carrier relaxation dynamics under electrical and weak optical injection. The physical process associated with the SHB recovery is likely Auger scattering from the excited states into the dot ground state, as indicated by the comparison of the recovery dynamics above and below transparency. This result excludes relaxation bottleneck problems in electrically pumped QD amplifiers.

$\mathrm{CH}$ is found to play a minor role in the gain compression recovery of the QD devices. A comparison with results on a commercial InGaAsP bulk amplifier shows that the $\mathrm{CH}$ in the dot devices is reduced compared with the bulk case, both in the gain and in the phase dynamics. Stimulated transitions lead to heating or cooling as observed via the phase dynamics that changes sign across transparency, and no significant free $\mathrm{CH}$ is found, different from what is typically observed in bulk and quantum-well devices. We believe that the free $\mathrm{CH}$ is inhibited in QD devices because of the smaller carrier density necessary to invert the dot ground-state transition compared with bulk and quantum-well devices. These results suggest superior performances of QD de- vices in high-speed applications compared with more conventional bulk and quantum-well devices.

\section{ACKNOWLEDGMENT}

The authors thank J. Mørk for many useful discussions and J. Bjørn at GIGA for mounting the samples. The authors acknowledge Tele Danmark R/\&D for the donation of part of the equipment.

\section{REFERENCES}

[1] D. Bimberg, M. Grundmann, and N. N. Ledentsov, Quantum Dot Heterostructures. New York: Wiley, 1998.

[2] N. N. Ledentsov, V. A. Shchukin, M. Grundmann, N. Kirstaedter, J. Böhrer, O. Schmidt, D. Bimberg, V. M. Ustinov, A. Yu. Egorov, A. E. Zhukov, P. S. Kop'ev, S. V. Zaitsev, N. Yu. Gordeev, Zh. I. Alferov, A. I. Borokov, A. O. Kosogov, S. S. Ruvimov, P. Werner, U. Gösele, and J. Heydenreich, "Direct formation of vertically coupled quantum dots in stranski-krastanov growth," Phys. Rev. B, vol. 54, pp. 8743-8750, 1996.

[3] F. Heinrichsdorff, M.-H. Mao, N. Kirstaedter, A. Krost, D. Bimberg, A. O. Kosogov, and P. Werner, "Room-temperature continuous-wave lasing from stacked InAs/GaAs quantum dots grown by metalorganic chemical vapor deposition," Appl. Phys. Lett., vol. 71, pp. 22-24, 1997.

[4] M.-H. Mao, F. Heinrichsdorff, A. Krost, and D. Bimberg, "Study of high frequency response of self-organized stacked quantum dot lasers at room temperature," Electron. Lett., vol. 33, pp. 1641-1642, 1997.

[5] K. Mukai, Y. Nakata, H. Shoji, M. Sugawara, K. Ohtsubo, N. Yokoyama, and $\mathrm{H}$. Ishikawa, "Lasing with low threshold current and high output power from columnar-shaped InAs/GaAs quantum dots," Electron. Lett., vol. 34, pp. 1588-1590, 1998.

[6] G. T. Liu, A. Stintz, H. Li, K. J. Malloy, and L. F. Lester, "Extremely low room-temperature threshold current density diode lasers using InAs dots in $\mathrm{In}_{0.15} \mathrm{Ga}_{0.85}$ As quantum wells," Electron. Lett., vol. 35, pp. $1163-1165,1999$.

[7] F. Schäfer, J. P. Reithmaier, and A. Forchel, "High-performance GaInAs/GaAs quantum-dot lasers based on a single active layer," Appl. Phys. Lett., vol. 74, pp. 2915-2917, 1999.

[8] J. Bloch, J. Shah, W. S. Hobson, J. Lopata, and S. N. G. Chu, "Room-temperature $1.3 \mu \mathrm{m}$ emission from InAs quantum dots grown by metal organic chemical vapor deposition," Appl. Phys. Lett., vol. 75, pp. 2199-2201, 1999.

[9] K. Nishi, H. Saito, S. Sugou, and J.-S. Lee, "A narrow photoluminescence linewidth of $21 \mathrm{meV}$ at $1.35 \mu \mathrm{m}$ from strain-reduced InAs quantum dots covered by $\mathrm{In}_{0.2} \mathrm{Ga}_{0.8}$ As grown on GaAs substrate," Appl. Phys. Lett., vol. 74, pp. 1111-1113, 1999.

[10] D. L. Huffaker, G. Park, Z. Zou, O. B. Shchekin, and D. G. Deppe, "1.3 $\mu \mathrm{m}$ room-temperature GaAs-based quantum-dot laser," Appl. Phys. Lett., vol. 73, pp. 2564-2566, 1998.

[11] A. E. Zhukov, A. R. Kovsh, N. A. Maleev, S. S. Mikhrin, V. M. Ustinov, A. F. Tsatsul'nikov, M. V. Maximov, B. V. Volovik, D. A. Bedarev, Yu. M. Shernyakov, P. S. Kop'ev, Zh. I. Alferov, N. N. Ledentsov, and D. Bimberg, "Long-wavelength lasing from multiply stacked InAs/InGaAs quantum dots on GaAs substrates," Appl. Phys. Lett., vol. 75, pp. 1926-1928, 1999.

[12] Yu. M. Shernyakov, E. Y. Kondrat'eva, D. A. Bedarev, P. S. Kop'ev, A. R. Kovsh, N. A. Maleev, M. V. Maximov, S. S. Mikhrin, A. F. Tsatsul'nikov, V. M. Ustinov, B. V. Volovik, A. E. Zhukov, Z. I. Alferov,

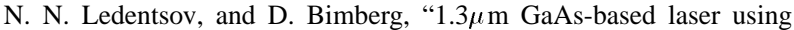
quantum dots obtained by activated spinodal decomposition," Electron. Lett., vol. 35, pp. 898-900, 1999.

[13] K. Mukai, Y. Nakata, K. Otsubo, M. Sugawara, N. Yokoyama, and H. Ishikawa, " $1.3 \mu \mathrm{m} \mathrm{CW}$ lasing of InGaAs-GaAs quantum dots at room temperature with a threshold current of $8 \mathrm{~mA}$," IEEE Photon. Technol. Lett., vol. 11, pp. 1205-1207, 1999.

[14] D. Bimberg, N. Kirstaedter, N. N. Ledentsov, Z. I. Alferov, P. S. Kop'ev, and V. M. Ustinov, "InGaAs-GaAs quantum-dot lasers," IEEE J. Select. Topics Quantum Elect., vol. 3, pp. 196-205, 1997.

[15] D. Klotzkin, K. Kamath, K. Vineberg, P. Bhattacharya, R. Murty, and J. Laskar, "Enhanced modulation bandwidth $(20 \mathrm{GHz})$ of $\mathrm{In}_{0.4} \mathrm{Ga}_{0.6} \mathrm{As} / \mathrm{GaAs}$ self-organized quantum dot lasers at cryogenic temperature: Role of carrier relaxation and differential gain," IEEE Photon. Technol. Lett., vol. 10, pp. 932-934, 1998. 
[16] T. S. Sosnowski, T. B. Norris, H. Jiang, J. Singh, K. Kamath, and P. Bhattacharya, "Rapid carrier relaxation in $\mathrm{In}_{0.4} \mathrm{Ga}_{0.6} \mathrm{As} / \mathrm{GaAs}$ quantum dots characterized by differential transmission spectroscopy," Phys. Rev B, vol. 57, pp. R9423-R9426, 1998.

[17] S. Raymond, X. Guo, J. L. Merz, and S. Fafard, "Excited-state radiative lifetime in self-assembled quantum dots obtained from state-filling spectroscopy," Phys. Rev. B, vol. 59, pp. 7624-7631, 1999.

[18] W. Yang, R. R. Lowe-Webb, H. Lee, and P. C. Sercel, "Effect of carrier emission and retrapping on luminescence time decays in InAs/GaAs quantum dots," Phys. Rev. B, vol. 56, pp. 13,314-13,320, 1997.

[19] R. Heitz, M. Veit, N. N. Ledentsov, A. Hoffmann, D. Bimberg, V. M Ustinov, P. S. Kop'ev, and Zh. I. Alferov, "Energy relaxation by multiphonon processes in InAs/GaAs quantum dots," Phys. Rev. B, vol. 56, pp. 10,435-10,445, 1997.

[20] C. T. Hultgren and E. P. Ippen, "Ultrafast refractive index dynamics in AlGaAs diode laser amplifiers," Appl. Phys. Lett., vol. 59, pp. 635-637, 1991.

[21] K. L. Hall, G. Lenz, E. P. Ippen, and G. Raybon, "Heterodyne pump-probe technique for time domain studies of optical nonlinearities in waveguides," Opt. Lett., vol. 17, pp. 874-876, 1992.

[22] J. Mark and J. Mørk, "Subpicosecond gain dynamics in InGaAsP optical amplifiers: Experiment and theory," Appl. Phys. Lett., vol. 61, pp. 2281-2283, 1992

[23] J. Mørk, J. Mark, and C. P. Seltzer, "Carrier heating in InGaAsP laser amplifiers due to two-photon absorption," Appl. Phys. Lett., vol. 64, pp. 2206-2208, 1994.

[24] K. L. Hall, G. Lenz, A. M. Darwish, and E. P. Ippen, "Subpicosecond gain and index nonlinearities in InGaAsP diode lasers," Opt. Commun., vol. 111, pp. 589-612, 1994.

[25] C.-K. Sun, B. Golubovic, H.-K. Choi, C. A. Wang, and J. G. Fujimoto, "Femtosecond investigations of spectral hole burning in semiconductor lasers," Appl. Phys. Lett., vol. 66, pp. 1650-1652, 1995.

[26] C.-K. Sun, B. Golubovic, J. G. Fujimoto, H. K. Choi, and C. A. Wang, "Heterodyne nondegenerate pump-probe measurement technique for guided-wave devices," Opt. Lett., vol. 20, pp. 210-212, 1995.

[27] J. Mørk, A. Mecozzi, and C. Hultgren, "Spectral effects in short pulse pump-probe measurements," Appl. Phys. Lett., vol. 68, p. 449, 1996.

[28] F. Heinrichsdorff, "MOCVD growth and laser applications of In(Ga)As/GaAs quantum dots," Ph.D. dissertation, Insitut für Festkörperphysik, Technische Universität Berlin, Germany, 1997.

[29] P. Borri, W. Langbein, J. Mørk, and J. M. Hvam, "Heterodyne pump-probe and four-wave mixing in semiconductor optical amplifiers using balanced lock-in detection," Opt. Commun., vol. 169, pp. 317-324, 1999

[30] A. Schönfelder, S. Weisser, J. D. Ralston, and J. Rosenzweig, "Differential gain, refractive index, and linewidth enhancement factor in high-speed GaAs-based MQW lasers: Influence of strain and p-doping," IEEE Photon. Technol. Lett., vol. 6, pp. 891-893, 1994.

[31] C. M. A. Kapteyn, F. Heinrichsdorff, O. Stier, R. Heitz, M. Grundmann, N. D. Zakharov, D. Bimberg, and P. Werner, "Electron escape from InAs quantum dots," Phys. Rev. B, vol. 60, pp. 14,265-14,268, 1999.

[32] J. Mørk and A. Mecozzi, "Theory of the ultrafast optical response of active semiconductor waveguides," J. Opt. Soc. Amer. B, vol. 13, pp. 1803-1816, 1996

[33] M. Grundmann and D. Bimberg, "Gain and treshold of quantum dot lasers: Theory and comparison to experiments," Jpn. J. Appl. Phys., vol 36, pp. 36-42, 1997.

[34] A. Mecozzi and J. Mørk, "Theory of heterodyne pump-probe experiments with femtosecond pulses," J. Opt. Soc. Amer. B, vol. 13, pp. 2437-2452, 1996

[35] R. Ferreira and G. Bastard, "Phononassisted capture and intradot auger relaxation in quantum dots," Appl. Phys. Lett., vol. 74, pp. 2818-2820, 1999.
[36] J. L. Pan, "Intraband auger processes and simple models of the ionization balance in semiconductor quantum-dot lasers," Phys. Rev. B, vol. 49, pp. 11,272-11,287, 1994

[37] P. Borri, W. Langbein, J. Mørk, J. M. Hvam, F. Heinrichsdorff, M.-H. Mao, and D. Bimberg, "Dephasing in InAs/GaAs quantum dots," Phys. Rev. $B$, vol. 60, pp. 7784-7787, 1999.

[38] P. Borri, W. Langbein, J. M. Hvam, F. Heinrichsdorff, M.-H. Mao, and D. Bimberg, "Time-resolved four-wave mixing in InAs/InGaAs quantum-dot amplifiers under electrical injection," Appl. Phys. Lett., vol. 76, pp. 1380-1382, 2000.

[39] M. Sugawara, K. Mukai, and Y. Nakata, "Light emission spectra of columnar-shaped self assembled InGaAs/GaAs quantum dot lasers: Effect of homogeneous broadening of the optical gain on lasing characteristics," Appl. Phys. Lett., vol. 74, pp. 1561-1563, 1999.

[40] D. Bimberg and J. Mycielski, "Recombination-induced heating of free carriers in a semiconductor," Phys. Rev. B, vol. 31, pp. 5490-5493, 1985.

P. Borri, (M'00) photograph and biography not available at the time of publication.

W. Langbein, photograph and biography not available at the time of publication.

J. M. Hvam, photograph and biography not available at the time of publication.

F. Heinrichsdorff, photograph and biography not available at the time of publication.

M.-H. Mao, photograph and biography not available at the time of publication.

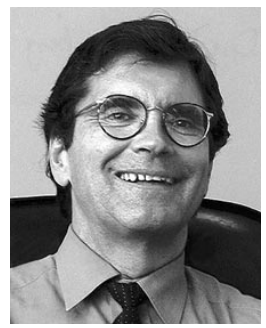

Dieter Bimberg (M'92) was born in Schrozberg, Germany, on July 10, 1942. He received the Diploma in physics and the Ph.D. degree from the Goethe University, Frankfurt, Germany, in 1968 and 1971, respectively.

From 1972 to 1979 he was a Senior Scientist with the Max Planck-Institute for Solid State Research, Stuttgart, Germany. From 1979 to 1981, he was an Associate Professor with the Department of Electrical Engineering, University of Aachen, Aachen, Germany. He presently holds the Chair of Applied Solid State Physics and is Director of the Solid State Physics Institute at the Technical University of Berlin, Berlin, Germany. He has authored more than 600 papers, patents, and books. His research interests include the physics of nanostructures and nanostructured devices, and high-speed photonic devices. He coordinates the DFB Center of Excellence on "Growth Related Properties of Nanostructures" and the national "Center of Competence on Nano-Optoelectronics." 